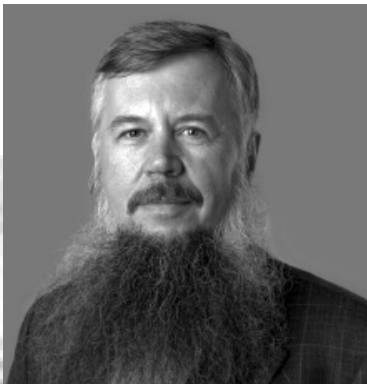

Prof. Dr. Gerhard Weber Technische Universität Dresden

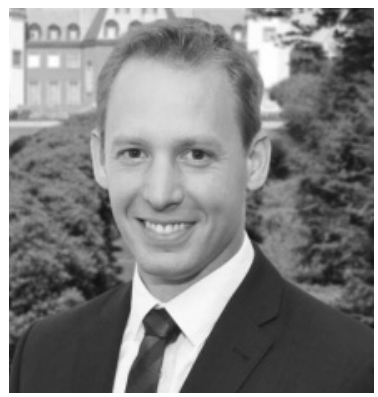

René Reiners

Fraunhofer-Institut für Angewandte Informationstechnik FIT Schloss Birlinghoven, Sankt Augustin

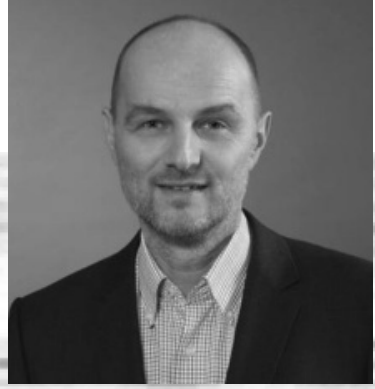

Prof. Dr.-Ing. Rainer Stiefelhagen Karlsruher Institut für Technologie (KIT)

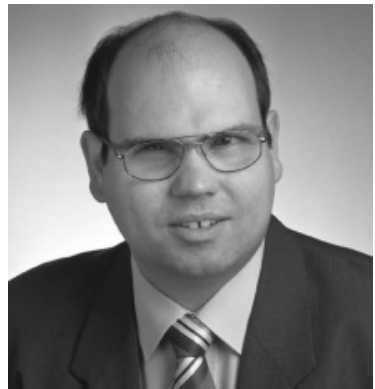

Dr. Steffen Puhl Justus-Liebig-Universität Gießen (Foto: Petra Pönnighaus-Martin)

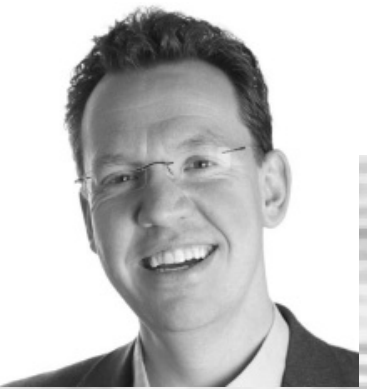

Prof. Dr. Ricardo Büttner Hochschule Aalen

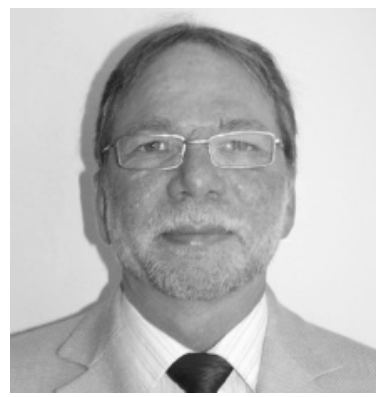

Henning Lübbecke Sprecher der Fachgruppe "Informatik und Inklusion", Bundesamt für Familie und zivilgesellschaftliche Aufgaben

\title{
Accessibility Beyond the Desktop
}

Die Teilhabe an der Informationsgesellschaft zu fördern, ist zu einem wichtigen gesellschaftlichen Auftrag für Informatiker geworden, der sich in der Gestaltung von Projekten aller Art widerspiegelt. Eine Reihe von Softwarehäusern und Internetagenturen haben Fachabteilungen gegründet bzw. Mitarbeiter darin geschult um Beratungen, entwicklungsbegleitende Dienstleistungen und die Evaluation der Barrierefreiheit von Benutzeroberflächen anzubieten. Damit wird Normalität was vor 25 Jahren noch schwer denkbar war: damals wurde von Wissenschaftlern die Pixel-Barriere als Forschungsaufgabe wahrgenommen. Gesucht wurde eine Möglichkeit, den Text in grafischen Darstellungen per Sprachsynthese oder Braille darzustellen. Die Pixel-Barriere bewirkte, dass blinde Menschen Zugang zu DesktopComputern verlieren. Durch diesen Paradigmenwechsel der Interaktionstechnik wurden Arbeitsplätze behinderter Menschen stark gefährdet. Geeignete Screenreader sind heute in Desktop-PCs integriert und die Pixel-Barriere kann bei richtiger Gestaltung der Benutzeroberfläche durch diese Software vermieden werden.

Heutige Forschungsprojekte zur Barrierefreiheit entwickeln für blinde Menschen u. a. die nicht-visuelle Interaktion mit Daten die per Computer Vision in einem beliebigen Kontext ermittelt werden. Brain-Computer Interfaces sind ein weiteres Beispiel, die helfen könnten bei schwerer Form von Autismus die Kommunikation zu ermöglichen. Andere Forschungsprojekte zielen auf die Schaffung einer adaptiven Infrastruktur ab, die nicht einzelne Behinderungen in den Mittelpunkt stellt, sondern für möglichst viele Formen von Behinderungen und damit für alle Benutzer im weitesten Sinn einen personalisierten, barrierefreien Zugang zu ganz alltäglichen Produkten wie Fahrkartenautomat, TV oder Webbrowser ohne besondere Vorkehrungen bieten.

Der Begriff Barrierefreiheit bezieht sich nicht mehr nur auf bauliche Maßnahmen für Rollstuhlfahrer sondern auch auf den Zugang zu Informationen am Arbeitsplatz, auf Dienstleistungen der öffentlichen Verwaltung und auf Lehrmaterialien in der Ausbildung. Architektur, Erziehungswissenschaften oder Arbeitspsychologie befassen sich seit langem mit Diversität und untersuchen das Zusammenspiel der Mechanismen die einzelne Akteure erbringen, um 


\section{\{EDITORIAL}

zur Inklusion beizutragen. Informatikern ist schnell klar, statt Insellösungen für Menschen mit einer Behinderung zu pflegen, ist es ökonomischer die Vielfalt unserer sensorischen, physischen oder kognitiven Fähigkeiten rechtzeitig im Entwicklungsprozess zu adressieren und geeignete Formen der Interaktion mit Computern zu entwickeln, die an diese Fähigkeiten angepasst sind.

Dies betrifft auch die Ausbildung von Informatikern, die Kenntnis und Umsetzung gesetzlicher Anforderungen im Rahmen von Pflichtenheften und Vereinbarungen mit dem Betriebsrat, aber auch zunehmend die Unterstützung anderer Fachabteilungen z. B. durch Entwicklung geeigneter Middleware für Betriebssysteme, oder für Webtechnologien. Ein wichtiges Etappenziel ist es born digital als born accessible Information zu verstehen, um die Teilhabe in der Informationsgesellschaft für eine möglichst breite Diversität zu verankern.

Dieses Heft gibt einen kleinen Einblick in aktuelle Forschung zu den speziellen Fähigkeiten von Menschen mit einer Behinderung, darauf aufbauende neue Interaktionstechniken und der Gestaltung von softwaretechnischen Schnittstellen für barrierefreie Benutzeroberflächen.

Der eingeladene Beitrag von Christian Bühler gibt in diesem Heft einen einführenden Überblick zu Gesetzen, Verordnungen und Richtlinien, um Barrierefreiheit näher zu beschreiben und festzulegen.

Accessibility Beyond the Desktop zielt auf die Teilhabe in einem vielfältigeren Umfeld, als es der Zugang zu Desktop-Computern ermöglicht und damit auch auf das private Umfeld. In einem Museum beispielsweise, sind gedruckte 3D Reliefs eine effiziente Möglichkeit haptische Interaktion zu ermöglichen, insbesondere wenn leitfähige oder transparente Druckmaterialien es erlauben die Berührung mit den Händen zu erkennen und damit blinden Menschen einen interaktiven Zugang zu Artefakten zu ermöglichen. Beiträge von Timo Götzelmann und Saiganesh Swaminathan et al. stellen den Einsatz von 3D Druckern für eine haptische bzw. audiotaktile Interaktion vor.

Unser Verständnis von nicht-visueller Präsentation ist noch gering und daher müssen am Körper getragene kleine Computer (Wearables) meist ein Display besitzen. Um die Verbesserung der Mobilität blinder Menschen durch taktile Wearables zu ermöglichen, untersucht der Beitrag von Limin Zeng Aspekte der kollaborativen Barrierefreiheit, einem noch jungen Gebiet innerhalb der Forschung zur Accessibility. Die Umsetzung der meist nicht-verbalen Aspekte der Kommunikation von blinden Menschen mit sehenden Menschen mittels nicht-visueller Interaktionstechniken und einem neuartigen Wearable untersuchen M. Saquib Sarfraz et al. in einem weiteren Beitrag.

Zur Adressierung kognitiver Behinderungen wurden bisher vor allem computerlinguistische Ansätze zur Verbesserung der Alternative Augmentative Communication (AAC) geschaffen. Der Beitrag von Dietmar Zoerner et al. beschäftigt sich mit der Anforderung an Menschen mit Autismus sich fehlende soziale Verhaltensweisen anzueignen und schlägt ein Spiel zum Erlernen der Erkennung von Emotionen mit adaptiver Trainingskomponente vor. Die Diagnose allgemeiner Lernschwierigkeiten im Rahmen einer neuartigen Lernplattform wird von Andreas Mühling vorgestellt. Die Lernverlaufsmessung lässt sich direkt im schulischen Unterricht einsetzen.

Drei Beiträge widmen sich der nicht-visuellen Interaktion mit grafischen Benutzungsoberflächen. Obwohl Screenreader längst auch in Mobiltelefonen (kostenlos) integriert sind, gilt es Grenzen im Umgang mit grafischen Notationen und letztlich beliebigen grafischen Darstellungen zu überwinden. Der Beitrag von Karin Müller et al. stellt einen neuartigen Ansatz für den Zugang zur UML Notation vor und Denise Bornschein et al. berichten über ein- und beidhändigen Gestaltungsformen der taktilen Interaktion mit berührempfindlichen Stiftplatten. Marlene Karlapp et al. haben eine dafür geeignete Softwareschnittstelle weiterentwickelt und betrachten die Flexibilität der Gestaltung der Benutzungsoberfläche von Screenreadern durch sehende Assistenten blinder Benutzer.

Eine spannende Lektüre wünschen

\section{Gerhard Weber, Rainer Stiefelhagen, Ricardo Büttner, René Reiners, Steffen Puhl, Henning Lübbecke}

